\title{
Segurança do paciente na hemotransfusão: uma revisão bibliométrica
}

\author{
Patient safety in hemotransfusion: a bibliometric review \\ Seguridad del paciente en hemotransfusión: una revisión bibliométrica
}

Recebido: 12/08/2021 | Revisado: 22/08/2021 | Aceito: 24/08/2021 | Publicado: 25/08/2021

\author{
Janice de Matos Frazão \\ ORCID: https://orcid.org/0000-0003-0621-5443 \\ Universidade do Estado do Pará, Brasil \\ E-mail: janice.dmfrazao@aluno.uepa.br \\ Edjane Marcia Linhares Melo \\ ORCID: https://orcid.org/0000-0001-5264-0139 \\ Universidade do Estado do Pará, Brasil \\ E-mail: edjanemelorx@gmail.com \\ Ivonete Vieira Pereira Peixoto \\ ORCID: https://orcid.org/0000-0002-5463-9630 \\ Universidade do Estado do Pará, Brasil \\ E-mail: ivonetep@hotmail.com \\ Rubenilson Caldas Valois \\ ORCID: https://orcid.org/0000-0001-9120-7741 \\ Universidade do Estado do Pará, Brasil \\ E-mail: rubenilsonvalois@ig.com.br
}

\begin{abstract}
Resumo
Esta pesquisa tem como objetivo identificar através dos estudos bibliométricos como se dá a produção cientifica sobre a segurança do paciente durante a hemotransfusão. Trata-se de uma revisão bibliométrica com uma abordagem descritiva, foi utilizado a base dados da Web of Science, e para análise descritiva utilizou-se o software VOSviewer, considerou-se os artigos publicados nos últimos cinco anos (2017-2021). O autor com maior número de artigos publicados foi Delaney, Meghan, com 19 artigos publicados e o mais recente é de 2020, o periódico com maior número de artigos foi a revista Transfusion com 54 publicações e a o termo "transfusion" foi caracterizado como o conceito central das publicações. Conclui-se que às análises relacionadas dos autores permitiram conhecer os autores que mais publicam artigos, os periódicos foram possíveis conhecer as suas produções e as análises das palavraschaves adquiridas pelo VOSviewer foi possível conhecer as principais relacionadas á temática exposta.

Palavras-chave: Transfusão de sangue; Enfermagem em hemoterapia; Estudos bibliométricos.
\end{abstract}

\begin{abstract}
This research aims to identify, through bibliometric studies, how the scientific production on patient safety during blood transfusion takes place. This is a bibliometric review with a descriptive approach, using the Web of Science database, and for descriptive analysis the VOSviewer software was used, considering the articles published in the last five years (2017-2021). The author with the highest number of articles published was Delaney, meghan, with 19 articles published and the most recent is from 2020, the journal with the highest number of articles was the journal Transfusion with 54 publications and the term "transfusion" was characterized as the central concept of publications. It is concluded that the related analyzes of the authors allowed us to know the authors who publish the most articles, the journals were able to know their productions and the analysis of the keywords acquired by VOSviewer made it possible to know the main ones related to the exposed theme.
\end{abstract}

Keywords: Blood transfusion; Hemotherapy nursing; Bibliometric studies.

\section{Resumen}

Esta investigación tiene como objetivo identificar, a través de estudios bibliométricos, cómo se produce la producción científica sobre la seguridad del paciente durante la transfusión de sangre. Se trata de una revisión bibliométrica con enfoque descriptivo, utilizando la base de datos Web of Science, y para el análisis descriptivo se utilizó el software VOSviewer, considerando los artículos publicados en los últimos cinco años (2017-2021). La autora con mayor número de artículos publicados fue Delaney, meghan, con 19 artículos publicados y el más reciente es de 2020, la revista con mayor número de artículos fue la revista Transfusion con 54 publicaciones y el término "transfusión" se caracterizó como el concepto central de publicaciones. Se concluye que los análisis relacionados de los autores permitieron conocer los autores que más artículos publican, las revistas pudieron conocer sus producciones y el análisis de las palabras clave adquiridas por VOSviewer permitió conocer las principales relacionadas con la tema expuesto.

Palabras clave: Transfusión de sangre; Enfermería en hemoterapia; Estudios bibliométricos. 


\section{Introdução}

A hemotransfusão é um método muito comum nas práticas assistenciais, pois não está livre de reações que podem surgir durante o processo de administração de sangue e de seus hemoderivados (Silva et al. 2017 \& Encan, 2019). A hemoterapia é caracterizada como uma área da medicina que aborda o emprego do sangue nos cuidados a saúde. Para realizar a transfusão de sangue em um indivíduo que esteja precisando é necessário que o médico prescreva e a enfermagem é responsável por todo o manejo com paciente que está recebendo um hemoderivado ou hemocomponente (Silva et al, 2018 \& Vilar et al. 2020). Sabe-se que apesar dos avanços tecnológicos, a utilização de novos testes e sorologias diferentes no momento da transfusão de sangue ainda corre o risco na transmissão de doenças do receptor para o doador, ou reações adversas dependendo do sistema imunológico do paciente (Reis \& Maia, 2020 \& Passerini, 2019).

Em alguns casos dependo da doença que o usuário apresenta a hemotransfusão torna-se indispensável, por isso, que a hemovigilância passou a ser mais rígida garantindo maior segurança ao usuário e seus familiares, para tal a transfusão de sangue e de seus hemoderivados é denominado como procedimento terapêutico, podendo salvar a vida de indivíduo doente (Silva et al. $2021 \&$ Langston, 2017). Nesse sentido, as principais reações transfusionais que os pacientes podem apresentar durante ou após a transfusão consiste em: reação alérgica, hemolítica aguadas e as febris (Reis et al. 2016 \& Cavalcante, 2016). Por isso, a segurança do paciente na hemotransfusão constitui em diminuir os riscos aos pacientes, a mesma deve ser apropriada as necessidades de cada paciente (Leite et al. 2018).

Para manter a segurança do paciente durante a execução da transfusão de sangue deve ser necessário conservar nos prontuários dos usuários sujeito a esta prática, os registros sobre a data, hora, início, término, temperatura, pressão arterial, e frequência cardíaca e possíveis reações que podem surgir (Ramos et al. 2017). Nos primeiros dez minutos o enfermeiro deve ficar beira leito para agir imediatamente caso ocorra possíveis intercorrência, promovendo assim a segurança do paciente durante todo esse procedimento transfusional (Mattia \& Andrade, 2016). Nesse contexto exige que os profissionais de enfermagem sejam habilitados para realizar essa técnica, visto que são os enfermeiros que executam o ato transfusional nos setores de saúde (Carneiro et al. 2017).

A segurança do paciente é fundamental para manter a qualidade na assistência. Nós dia atuais vem ganhando maior destaque para os gestores, instituições e para os profissionais da área da saúde, possibilitando uma assistência livre de riscos, segura e eficaz para os pacientes e seus familiares diminuindo os possíveis riscos durante a assistência ofertada (Leite et al. 2018).

Tem como objetivo Identificar através dos estudos bibliométricos como se dá a produção cientifica sobre a segurança do paciente durante a hemotransfusão.

\section{Metodologia}

Para o desenvolvimento deste estudo optou-se em realizar uma pesquisa de revisão bibliométrica, com abordagem descritiva. Inicialmente foi acessado o portal de periódicos CAPES para proceder a escolha das bases de dados onde seriam coletados os artigos científicos de interesse de estudos. Assim a base de dados selecionada foi a Web of Science. A pesquisa foi realizada no mês de junho de 2020. Foram utilizadas as seguintes palavras-chave em inglês: blood transfusion, hemotherapy nursing e bibliometric studies e foram utilizados os operadores boleanos de truncamento "OR" e "END" entre os termos. Considerou-se artigos publicados dos últimos cinco anos (2017-2021) e a análise de dados foi realizada a partir do software VOSviewer. consolidou-se em 2.560 artigos cientificos, correspondendo ao corpo de análise.

O estudo bibliométrico consiste em realizar uma análise estatística de aspecto quantitativo, sobre as produções cientificas (Barros et al. 2020). Assim pode ajudar a identificar o progresso de uma determinada área de conhecimento, a reconhecer os autores, aos periódicos e as propagações das pesquisas em um determinado campo ou área de conhecimento, ou 
seja, as técnicas bibliométricas são pertinentes para a evidenciação dos trabalhos científicos realizados pelos pesquisadores (Soares et al. 2016).

Para realizar uma pesquisa bibliométrica é necessário conhecer as três leis relevantes da bibliométria, a primeira é chamada a lei de Lotka está relacionada a produtividade dos autores em determinado campo, a lei de Bradford está associada a produção de artigos científicos em periódicos, já a lei de Zipf refere-se quantas vezes as palavras-chave surgem em uma produção cientifica (Quevedo-Silva et al. 2016).

\section{Resultados e Discussão}

Tabela 1. Países com mais artigos publicados no período 2017-2021.

\begin{tabular}{l|l|c|l}
\hline Posição & Países & Autorias de artigos & \% do total dos artigos \\
\hline 1 & Brasil & 92 & 10,12 \\
2 & Inglaterra & 86 & 9,46 \\
3 & Singapura & 75 & 8,25 \\
4 & Áustria & 66 & 7,26 \\
5 & França & 65 & 7,15 \\
6 & Austrália & 50 & 5,5 \\
7 & Indonesia & 48 & 5,28 \\
8 & Canadá & 44 & 4,84 \\
9 & Israel & 28 & 3,08 \\
10 & Japão & 27 & 2,97 \\
11 & Países Baixos & 15 & 1,65 \\
\hline
\end{tabular}

Fonte: Frazão (2021).

Foram selecionados 11 países que mais publicaram artigos de acordo com a bases de dados da Web Of Science, sendo o Brasil com o maior índice de autorias de artigos em relação a segurança do paciente na hemotranfusão com 92 das autorias correspondendo $(10,12 \%)$ do total das publicações. A Holanda denominada pelo nome Paises Baixos, apresentou 15 das autorias correpondendo $(1,65 \%)$, foi cararcterizada como o país com o menor número de autorias em relação a segurança do paciente na hemotransfusão.

Tabela 2. Autores com mais artigos publicados no período 2017-2021.

\begin{tabular}{l|l|c|l}
\hline Posição & Autor & Artigos & \% do total dos artigos \\
\hline 1 & Delaney, meghan & 19 & 2,09 \\
2 & Haspel, Richard I & 15 & 1,65 \\
3 & Luban, Naomi I. C & 12 & 1,32 \\
4 & Goel, Ruchika & 11 & 1,2 \\
5 & Karam, Oliver & 09 & 0,99 \\
6 & Nellis, Mariane E & 08 & 0,88 \\
7 & Josephson, Cassandra & 05 & 0,55 \\
8 & Solar-Visner, Martha & 04 & 0,44 \\
\hline
\end{tabular}

Fonte: Frazão (2021). 
O autor com maior número de artigos publicados foi Delaney, meghan, com 19 artigos publicados e o mais recente é de 2020. O segundo autor com mais publicações é Haspel, Richard I com 15 registros e o Luban, Naomi I. C com 12 documentos. Na sequência Goel, Ruchika com 11 publicações, Karam, Oliver com 09 artigos, Nellis, Mariane E com 08 artigos, Josephson, Cassandra D com 05 registros e Solar-Visner, Martha com 04 artigos publicados.

Tabela 3. Periódicos com mais artigos publicados no período 2017-2021.

\begin{tabular}{l|l|c|l}
\hline Posição & Periódicos & Artigos & $\%$ \\
\hline 1 & Transfusion & 54 & 5,94 \\
2 & Clinical Transplantation & 48 & 5,28 \\
3 & Hemoglobin & 32 & 3,52 \\
4 & Annals of Palliative Medicine & 28 & 3,08 \\
5 & Acta Cardiologica & 27 & 9,97 \\
6 & A \& A Practice & 24 & 2,64 \\
7 & American Journal of Case Reports & 20 & 2,2 \\
8 & Journal of Clinical Laboratory Analysis & 17 & 1,87 \\
9 & Internal Medicine Journal & 15 & 1,65 \\
10 & Langenbecks Archives of Surgery & 13 & 1,43 \\
\hline
\end{tabular}

Fonte: Frazão (2021).

A lista contém um ranking com 10 posições, O periódico com maior número de artigos foi a revista Transfusion com 54 publicações. Na segunda posição a revista Clinical Transplantation com 48 publicações e na terceira posição a o periodico Hemoglobin com 32 artigos publicados. Sendo assim a sequência consiste Annals of Palliative Medicine com 28 artigos, Acta Cardiologica com 27 artigos, A \& A Practice com 24 artigos, American Journal of Case Reports com 24 artigos, Journal of Clinical Laboratory Analysis com 17 artigos, Internal Medicine Journal com 15 artigos, Langenbecks Archives of Surgery com 13 artigos publicados. É importante destacar que os periódicos são das áreas da saúde. 
Figura 1. Mapa das palavras-chave dos autores no período 2017-2021.

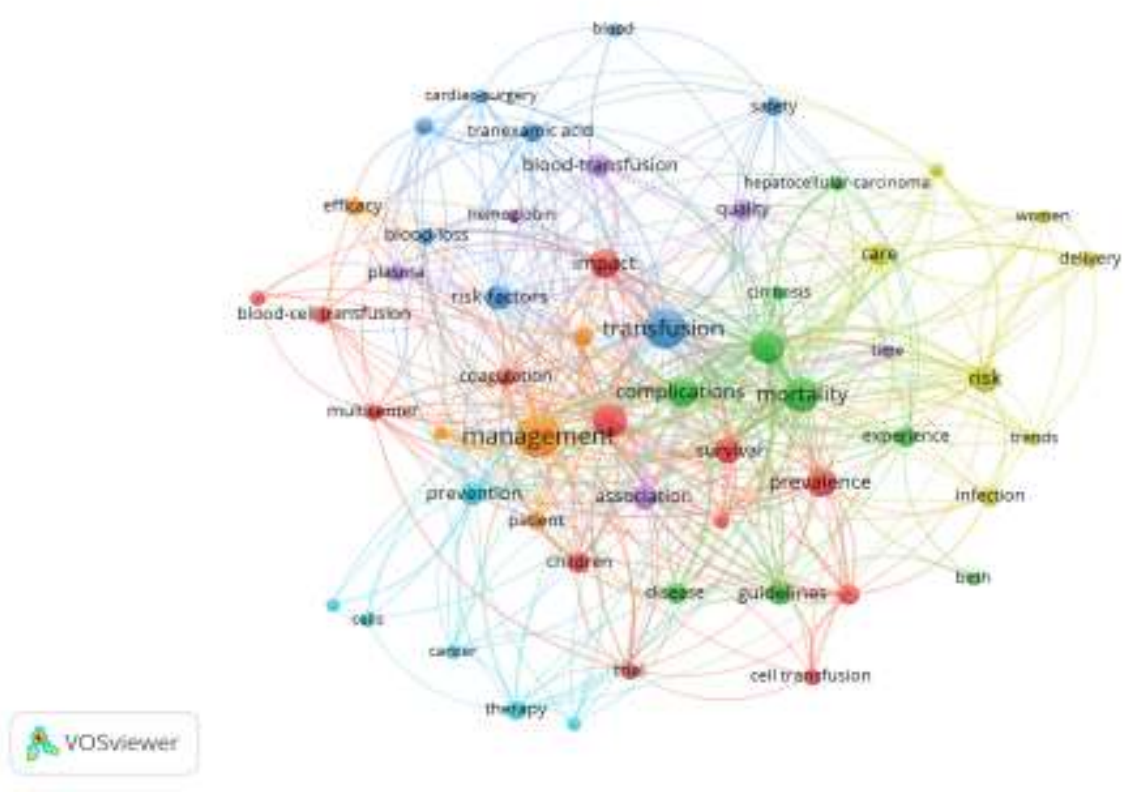

Fonte: Frazão e aplicativo VOSviewer (2021).

O mapa destaca o termo "transfusion" como sendo o conceito central de todas as relações estabelecidas. Dentre os outros conceitos que possuem fortes ligações com os demais e podem ser destacados encontram-se "managment", “complications", “outcomes", “mortality”, “surgery” e "risk”. O VOSviewer organizou os elementos do mapa em 07 clusters com 57 itens. O maior deles contém 13 itens e é representado pela cor vermelha no mapa abriga itens como "anemia”, "bloodcell transfusion", “children”, “coagulation”, “impact”, “metaanalysis”, “multicenter”, "prevalence”, “surgery”, “survival”, "trauma", "trial". O segundo maior cluster, representado em verde no mapa, contém 09 itens "birth", "cirrhosis", “complications", “disease", “experience", "guidelines”, "hepatocelular-carcion”, "mortality”, “outcomes”. O terceiro cluster, representado em azul no mapa, contém 08 itens "blood”, "blood-loss", “cardiac-surgery", “double-blind”, "risk-factors", "safety", "tranexamic acid", "transfusion". O quarto cluster, contém 07 itens “care”, “delivery", "infection”, “pregnancy”, "risk", "trends", “womwn”. O quinto cluster, contém 06 itens "association”, "blood-trnasfusion”, "hemoglobin”, “plasma”, “quality”, “time”. O sexto cluster, contém 06 itens “câncer”, “cells”, “diagnosis”, "prevention”, “storage”, "therapy” e o sétimo cluster, contém 05 itens “efficacy", “management", "patient”, “predictors", "replacement".

\section{Conclusão}

Conclui-se que a partir desse trabalho foi possível conhecer um pouco mais sobre o campo da bibliométria na área da saúde. As análises relacionadas dos autores permitiram conhecer os autores que mais publicam artigos. Já os periódicos foram possíveis agrupar e conhecer as suas produções. Por fim com as análises das palavras-chaves adquiridas por meio do VOSviewer foi possível conhecer as principais relacionadas á temática exposta. É importante salientar que o tema abordado é relevante para área acadêmica.

\section{Referências}

Carneiro, V. S. M., Barp, M., \& Coelho, M. A. (2017). Hemoterapia e reações transfusionais imediatas: atuação e conhecimento de uma equipe de enfermagem. Rev Min Enferm, 21, 1031.

Leite, G. R., Assis, C. L, Freitas, G. S. I, Maia, L. G, Eid, L. P, Martins, M. A, Paulino, V. C. P., \& Sthall, H. C. (2018). Segurança do paciente na hemotransfusão: atitude e conhecimento de enfermeiros no sudoeste de Goiás. Revista eletrônica graduação/pós-graduação em educação, 14 (4), 1807-9342. 
Mattia, D. D, \& Andrade, S. R. D. (2016). Cuidados de enfermagem na transfusão de sangue: um instrumento para monitorização do paciente. Texto Contexto Enferm, 25 (2), 2600015.

Ramos, P. D. S, Amorim, V. C, Ferreira, C. B. T, Romaneli, D. A. V. D. R, Campos, I. M., \& Dias, V. L. (2017). Reação hemolítica transfusional: diagnóstico e manejo anestésico. Rev Med Minas Gerais, 27 (4), S46-S51.

Reis, V. N. D, Paixa, I. B, Perrone, A. C. A. D. J, Monteiro, M. I., \& Santos, K. B. D. 2016. Monitorização transfusional: análise da prática assistencial em um hospital público de ensino. Einstein, 14 (1), 41-6.

Silva, J. D. B, Sabino, K. C. V, Brito, L. V. B. S. D, Silva, A. M. D, Costa, E. D. S, Brito, A. L. D. S, Morais, F. J. D, Costa, K. R. D. A. L., \& Oliveira, G. C. 2018. As hemotransfusões e atuação do enfermeiro nos procedimentos alternativos. Brazilian Journal of Surgery and Clinical Research - BJSCR, 23 (1), 100105 .

Silva, P. A. R. D, Assis, D. C. M. D., \& Silva, C. R. D. 2017. Conhecimento de profissionais de enfermagem sobre atuação em hemotransfusão. Rev Ciên Saúde, 2 (2), 15-24

Encan. B., \& Akin, S. (2019). Knowledge of Blood Transfusion Among Nurses. J Contin Educ Nurs, 50 (4), $176-182$.

Langston, A, Downing, D, Packard, J, Burcie, S., \& Lewis, B. (2017). Massive Transfusion Protocol Simulation An Innovative Approach to Team Training. Crit Care Nurs Clin N Am, 29, 259-269.

Passerini, H. M. (2019). Contemporary transfusion science and challenges. AACN Adv Crit Care. 30 (2), 139-150.

Silva, E. M. D, Vieira, C. A, Silva, F. D. O, Ferreira, E. V. (2017). Desafios da enfermagem diante das reações transfusionais. Rev enferm UERJ, $25,11552$.

Silva, A. T, Alves. M. G. Sanches. R. S, Terra, F. D. S., \& Resck, Z. M. R. (2016). Assistência de enfermagem e o enfoque da segurança do paciente no cenário brasileiro. Saúde Debate, 40 (111), 292-301.

Santos, D. R, Araújo, P. E., \& Silva, W. D. S. (2017). Segurança do paciente: uma abordagem acerca da atuação da equipe de enfermagem na unidade hospitalar. Temas em saúde. 17 (2), 213-225.

Cavalcante, A. K. C. B. (2016). Cultura de segurança na percepção da enfermagem: revisão integrativa. Rev. Enferm UFPE. 10 (10). $3890-7$.

Quevedo-Silva. F, Santos. E. B. A. Brandão. M. M., \& Vils. L. (2016). Estudo Bibliométrico: Orientações sobre sua Aplicação. Revista Brasileira de Marketing - ReMark, 15 (2), 246-262.

Soares, P. B, Carneiro, T. C. J, Calmon, J. L., \& Castro, L. O. D. C.O. (2016). Análise bibliométrica da produção científica brasileira sobre Tecnologia de Construção e Edificações na base de dados Web of Science. Ambiente Construído. 16 (1), 175-185.

Barros, A. V. M. D, Nascimento, V. H. S. D, Silva, C. C. G, Amaral, B. B. do, Cartaxo, R. D. O., \& Sette de-Souza, P. H. (2020). Levantamento bibliométrico dos artigos publicados na Revista da ABENO no período entre 2001 e 2019. Revista da ABENO, 20(2), 38-46.

Vilar, V. M, Ferreira, N. D. C, Nakasato, G. R. Lupinacci, F. L, Lopes J. D. L., \& Lopes, C. T. (2020). Fatores associados a reações transfusionais imediatas em um hemocentro universitário: estudo analítico retrospectivo. Revista Medicina, 53(3), 275-82.

Silva, D. O. D, Abreu. B. M. D, Medeiros, G. G. D. (2021). Enfermagem diante d a transfusão de sangue em pacientes testemunhas de jeová: ética e procedimentos alternativos. Rev Inic Cient Ext, 4(1), 562-77.

Reis, G. A. B., \& Maia, L. F D. S. (2020). Ética e bioética no contexto da recusa a transfusão sanguínea: revisando a literatura. Revista Recien, 10 (29), 137144. 